\title{
Increased Resting State Functional Connectivity in the Default Mode Network in Recovered Anorexia Nervosa
}

\author{
Felicity A. Cowdrey, 'Nicola Filippini,, ${ }^{1,2}$ Rebecca J. Park,' \\ Stephen M. Smith, ${ }^{2}$ and Ciara McCabe ${ }^{1 *}$ \\ ${ }^{1}$ University of Oxford Department of Psychiatry, Warneford Hospital, Oxford, United Kingdom \\ ${ }^{2}$ University of Oxford FMRIB Centre, Nuffield Department of Clinical Neurosciences, \\ John Radcliffe Hospital, Headington, Oxford, United Kingdom
}

\begin{abstract}
Functional brain imaging studies have shown abnormal neural activity in individuals recovered from anorexia nervosa (AN) during both cognitive and emotional task paradigms. It has been suggested that this abnormal activity which persists into recovery might underpin the neurobiology of the disorder and constitute a neural biomarker for AN. However, no study to date has assessed functional changes in neural networks in the absence of task-induced activity in those recovered from AN. Therefore, the aim of this study was to investigate whole brain resting state functional connectivity in nonmedicated women recovered from anorexia nervosa. Functional magnetic resonance imaging scans were obtained from 16 nonmedicated participants recovered from anorexia nervosa and 15 healthy control participants. Independent component analysis revealed functionally relevant resting state networks. Dual regression analysis revealed increased temporal correlation (coherence) in the default mode network $(\mathrm{DMN})$ which is thought to be involved in self-referential processing. Specifically, compared to healthy control participants the recovered anorexia nervosa participants showed increased temporal coherence between the DMN and the precuneus and the dorsolateral prefrontal cortex/inferior frontal gyrus. The findings support the view that dysfunction in resting state functional connectivity in regions involved in self-referential processing and cognitive control might be a vulnerability marker for the development of anorexia nervosa. Hum Brain Mapp 35:483-491, 2014. (c) 2012 Wiley Periodicals, Inc.
\end{abstract}

Key words: resting state; functional connectivity; fMRI; anorexia nervosa; default mode network

\section{INTRODUCTION}

Anorexia nervosa (AN) is characterized by preoccupation with control of eating, weight, and shape leading to severe dietary restriction and weight loss to less than $85 \%$

Contract grant sponsor: Sir Jules Thorn Charitable Trust.

*Correspondence to: Ciara McCabe, Department of Psychiatry, University of Oxford, Warneford Hospital, Oxford OX3 7JX, United Kingdom. E-mail: ciara.mccabe@psych.ox.ac.uk.

Received for publication 21 March 2012; Revised 6 July 2012; Accepted 21 August 2012

DOI: $10.1002 / \mathrm{hbm} .22202$

Published online 3 October 2012 in Wiley Online Library (wileyonlinelibrary.com). of ideal body weight [American Psychiatric Association, 1994]. Individuals with $A N$ have an intense fear of gaining weight and/or becoming fat and a maladaptive system for judging self-worth which is often exclusively in terms of shape and weight and the ability to control them [American Psychiatric Association, 1994]. There is no clear evidence-based treatment for AN [Fairburn, 2005] and the disorder continues to have the highest mortality rate of all psychiatric disorders [Arcelus et al., 2011; Sullivan, 1995].

Advances in neuroimaging techniques have enabled investigation of the pathophysiology of AN and has led to the development of neurobiological frameworks for the disorder. For example, it has been hypothesized that in people with AN, hyperactivity of cognitive pathways (e.g., dorsolateral prefrontal cortex to dorsal striatum) direct 
motivated actions when the ability of ventral striatal pathways to direct more "automatic" or intuitive responses is impaired [Kaye et al., 2009]. The theoretical frameworks of Kaye et al. [2009] and that of Park et al. [2011, 2012], suggest ruminative preoccupation with control of eating, weight and shape may be central to the psychopathology of AN. These recent frameworks may help to explain some of the most complex features of AN, such as increased cognitive control over food and rumination on the control of eating and the body, which are unlikely to be explained by functional or structural breakdown in a single brain area.

Resting state functional connectivity is an fMRI approach that utilizes spontaneous fluctuations in the resting brain, enabling temporal correlations between brain areas to be mapped [Biswal et al., 2010; Greicius et al., 2009]. Brain regions showing a strong temporal coherence (spontaneous coactivation) are termed "resting state networks" (RSNs) and are thought to reflect intrinsic properties of functional brain organization [Damoiseaux et al., 2006; Gusnard and Raichle, 2001]. For example, the default mode network (DMN) that encompasses brain regions including the posterior cingulate, the precuneus and parts of the prefrontal cortex, is more active at rest than during attention-demanding tasks and is therefore thought to be associated with stimulus-independent thought and selfreflection [Raichle et al., 2001].

Although there is no data on resting state functional connectivity in AN, it has been examined in other neuropsychiatric disorders including depression, bipolar disorder, and schizophrenia [Greicius, 2008]. Increased functional connectivity has been found in the DMN in depressed patients highlighting a potential neural mechanism for the exaggerated negative self-focus that characterizes the disorder [Sheline et al., 2010; Veer et al., 2010]. Increased functional connectivity has also been shown to correlate with self-reported levels of ruminative brooding in depressed participants [Berman et al., 2010; Hamilton et al., 2010]. Importantly, it has also been shown that current psychoactive treatments can modulate resting state functional connectivity in healthy volunteers and patients, thus demonstrating a mechanism by which psychoactive medications may be having their therapeutic effects [Anand et al., 2005; McCabe and Mishor, 2011; McCabe et al., 2011].

To date, there have been no studies examining the neural networks at rest in AN and so hypotheses regarding the role of different neural circuits in AN have relied primarily upon inferences from task-induced activity in functional magnetic resonance imaging (fMRI) studies. For example, Uher et al. [2004] used a symptom-provoking fMRI paradigm and demonstrated that individuals with AN had increased activation in medial prefrontal regions as well as the anterior cingulate compared to healthy controls. Another study examined the neural correlates of cognitive and behavioral flexibility in $\mathrm{AN}$ and reported that impaired behavioral response shifting in AN was associ- ated with reduced activation in areas involved in motivation-related behavior and increased activation in brain regions involved in cognitive control during task performance [Zastrow et al., 2009].

Studying those currently affected by AN has its limitations as current malnutrition may cause widespread alterations in brain structure and function [Kaye et al., 2009]. It is therefore difficult to determine from these studies whether abnormalities in brain activations are state factors or whether they represent biomarkers. An alternative research design is to examine individuals recovered from $\mathrm{AN}$, as they continue to display not only temperamental and personality traits but neural aberrancies that may have predisposed them to the illness [Cowdrey et al., 2011; Jacobs et al., 2009; Wagner et al., 2006a]. For example, Wagner et al. [2007] showed that women recovered from AN had greater neural response to both positive and negative feedback in the caudate and dorsolateral prefrontal cortex; regions of the brain thought to be involved in higher level cognitive processes. The authors propose that this may represent increased cognitive control even after recovery from AN [Wagner et al., 2007].

Taken together these findings suggest that there may be alterations in resting state functional connectivity in AN, in networks modulating cognitive control and rumination which is consistent with the symptomatology of the disorder. Importantly, identifying neural network dysfunction in those recovered from AN might aid the discovery of neural biomarkers for this disorder. The aim of the present investigation was therefore to examine neural networks using resting state functional connectivity in women recovered from AN compared to healthy controls. As this is the first study on functional connectivity in this population (ill or recovered), we investigated whole brain functional connectivity rather than focusing on predefined regions of interest.

On the basis of the current neurobiological and theoretical frameworks for AN [Kaye et al., 2009; Park et al., 2011, 2012], and previous task-based fMRI studies [Cowdrey et al., 2011; Uher et al., 2004; Wagner et al., 2007], we hypothesized that altered connectivity would be seen in networks that encompass brain regions underpinning selfreferential processing (rumination) and cognitive control such as the DMN and the cognitive control network [Kaye et al., 2009; Park et al., 2011, 2012; van Kuyck et al., 2009].

\section{MATERIALS AND METHODS}

\section{Participants}

Sixteen women who had previously met DSM-IV criteria for AN and 15 healthy female controls were recruited for this study. Participants were recruited through web, e-mail, and poster advertisements. Ethical approval for the study was obtained from the University of Oxford and Outer West London Research Ethics Committee and 
written informed consent was obtained for all participants after complete description of the study.

All participants underwent a screening process which involved (a) a brief email screening (b) a face-to face assessment of psychiatric disorder using the Structured Clinical Interview for DSM-IV [Spitzer et al., 2004]. Current eating disorder symptoms in all participants were assessed using the Eating Disorder Examination-questionnaire (EDE-Q) [Fairburn and Beglin, 2008]. All participants completed a questionnaire battery which included: Beck Depression Inventory (BDI-II) [Beck et al., 2002], State Trait Anxiety Inventory (STAI) [Spielberger, 1983], Positive and Negative Affect Schedule [Watson et al., 1988], and Ruminative Response Scale for Eating Disorders (RRS-ED) [Cowdrey and Park, 2011] approximately 1 week before scanning. Current BMI, lowest ever BMI and duration of recovery was also recorded for participants in the recovered AN group.

Criteria for inclusion in the recovered group included (a) a history of DSM-IV AN, (b) maintenance of a BMI of between 18.5 and $25 \mathrm{~kg} / \mathrm{m}^{2}$ for at least 12 months prior to the study, (c) regular menstruation for at least 12 months prior to the study, (d) no use of psychoactive medications, such as antidepressants, for at least12 months prior to the study. In addition, recovered AN participants had to score within one standard deviation of the EDE-Q global mean scores for young women. All 16 recovered individuals had met criteria for restricting- type AN. In addition, four participants met criteria for bulimia nervosa and one met criteria for eating disorder not otherwise specified during their lifetime. Nine of the AN recovered participants fulfilled the criteria for major depressive disorder during their lifetime and 3 participants had fulfilled the criteria for obsessive compulsive disorder.

Inclusion criteria for the healthy control group were (a) BMI between 18.5 and $25 \mathrm{~kg} / \mathrm{m}^{2}$, (b) no first degree relative with a current or past eating disorder diagnosis, (c) no lifetime history of any Axis 1 psychiatric disorder on the Structured Clinical Interview for DSM-IV [Spitzer et al., 2004], (d) maintained a weight in the healthy range (defined by the World Health Organization) since menarche.

General exclusion criteria for all participants included a history of head injury, neurological or other severe medical illness, pregnancy and any contradictions to MRI. All participants were fluent in English, right handed, had normal or corrected to normal vision and were not taking medication except for the contraceptive pill.

\section{Data Acquisition}

Imaging data were acquired using Sieman's Avanto 1.5-T whole body scanner at the Oxford Centre for Magnetic Resonance Imaging. Resting state fMRI data were acquired after a 10-min no-task anatomical scan. Before this the participants completed two task-related MRI runs [Cowdrey et al., 2011, 2012]. Participants were instructed to lie in dimmed light with their eyes open, think of nothing in particular, and not to fall asleep. T2*-weighted EPI slices were acquired every 3 $\mathrm{s}(\mathrm{TR}=3)$. Axial slices (35) with in-plane resolution of $3 \mathrm{~mm}$ $\times 3 \mathrm{~mm}$ and between plane spacing of $4 \mathrm{~mm}$ were obtained. The matrix size was $64 \times 64$ and the field of view was 192 $\mathrm{mm} \times 192 \mathrm{~mm}$. Acquisition was carried out during the resting scan yielding 140 volumes in total (acquisition time $=5$ min). An anatomical T1-weighted sequence with axial plane slice, thickness $1 \mathrm{~mm}$, and in-plane resolution of $1.0 \mathrm{~mm} \times$ $1.0 \mathrm{~mm}$ was also acquired to improve the registration process and also for gray matter analysis.

\section{Resting State Analysis}

fMRI analysis was carried out using FSL [Jenkinson et al., 2011] with resting state networks identified using the independent component analysis (ICA) tool MELODIC (Multivariate Exploratory Linear Optimized Decomposition into Independent Components) [Beckmann et al., 2005]. Individual prestatistical processing consisted of motion correction, brain extraction, spatial smoothing using a Gaussian kernel of full-width at half-maximum 6 $\mathrm{mm}$, and high-pass temporal filtering equivalent to $150 \mathrm{~s}$ $(0.007 \mathrm{~Hz})$. fMRI volumes were registered to the individual's structural scan and standard space images using FMRIB's Nonlinear Image Registration Tool (FNIRT).

Preprocessed functional data containing 140 time-points for each subject were temporally concatenated across subjects, creating a single $4 \mathrm{D}$ dataset, to carry out group-wise ICA. Group-ICA was carried out at a dimensionality of 60 . RSNs of interest covered the entire brain and were selected using spatial correlation against sets of previously defined maps [Beckmann et al., 2005].

The subject-specific analysis of the resting data was carried out using a regression technique ("dual regression") [Filippini et al., 2009] that allows for voxel-wise comparisons of resting functional connectivity between subjects or subject groups. This approach proceeds in three stages: first, the (groupwise) concatenated multiple fMRI data sets are decomposed using ICA to identify large-scale patterns of functional connectivity in the population of subjects (as described earlier). Second, dual regression approach is used to identify, within each subject's fMRI data set, subject-specific temporal dynamics and spatial maps that are associated with each group IC map. This involves (A) using the full set of group-ICA spatial maps in a linear model fit (spatial regression) against the separate fMRI data sets, resulting in matrices describing temporal dynamics for each component for each subject and (B) using these subject-specific time-course matrices in a linear model fit (temporal regression) against the associated fMRI data set to estimate subject-specific spatial maps. Finally, the different component maps are collected across subjects into single $4 \mathrm{D}$ files (one per original ICA map, with the 4th dimension being subject ID) and tested voxelwise for statistically significant differences between the groups using nonparametric permutation testing $(5,000$ permutations) [Nichols and Holmes, 2002]. Clusters were 
determined by using threshold-free cluster enhancement (TFCE) [Smith and Nichols, 2009] and a family-wise error (FWE) corrected cluster significance threshold of $P=0.05$. This results in spatial maps characterizing the betweensubject/group differences. Nonparametric tests were used (10,000 permutations), to safeguard against the possibility that the between-subjects effects were non-Gaussian, and because such nonparametric inference has greater robustness against spatial nonstationarity than commonly used parametric methods [Hayasaka et al., 2004].

\section{Gray Matter Morphology}

Abnormalities in gray matter volume have been found previously in those recovered from AN [Joos et al., 2011; Katzman et al., 1997; Roberto et al., 2011] although this finding is not always reported [Castro-Fornieles et al., 2009; Wagner et al., 2006b]. To test whether altered functional connectivity in this study may be explained by differences between the groups in gray matter volume, voxelbased morphometry (VBM) analysis was run on the highresolution T1-weighted data sets [Douaud et al., 2007]. In brief, brain extraction and tissue-type segmentation were performed, and resulting GM partial volume images were aligned to standard space using FMRIB's Linear Image Registration Tool (FLIRT) and then nonlinear (FNIRT) registration tools. The resulting images were averaged, modulated to correct for nonlinear warp-field expansion, and smoothed with an isotropic Gaussian kernel of full width at half maximum $4 \mathrm{~mm}$ to create a study-specific template. Finally, voxel-wise GLM was applied using permutation nonparametric testing (5,000 permutations), correcting for multiple comparisons across space.

\section{RESULTS}

\section{Demographic Details and Mood Ratings}

Because of technical difficulties, only data from 14 of the 15 healthy control participants was included in the analysis. There were no significant differences between the two groups as determined by independent samples $t$-test for age, body mass index or IQ $(P>0.05)$ (Table I). MannWhitney $U$ tests were used to analyze data from the selfreport questionnaires. There were no significant differences between the groups for measures of eating disorder symptoms, depression, state or trait anxiety, and positive affect or negative affect $(P>0.05)$. However, the recovered AN group scored significantly higher than the healthy controls in terms of rumination on control of eating and body shape and weight $(P<0.05)$ (Table I).

\section{Resting State Functional Connectivity}

Independent component analysis (ICA) defined 60 independent components. Of these, 12 components were identified as RSNs (covering the majority of gray matter) and
TABLE I. Sociodemographic and mood ratings for the two groups

\begin{tabular}{lccc}
\hline & \multicolumn{2}{c}{ Mean $(S D)$} & \\
\cline { 2 - 3 } & $\begin{array}{c}\text { Recovered } \\
\text { AN }(n=16)\end{array}$ & $\begin{array}{c}\text { Healthy } \\
\text { controls }(n=14)\end{array}$ & $\begin{array}{c}P \text { (two } \\
\text { tailed) }\end{array}$ \\
\hline Age (years) & $23.06(3.55)$ & $24.11(2.85)$ & 0.38 \\
BMI & $21.33(2.17)$ & $21.01(1.56)$ & 0.65 \\
IQ & $115.93(4.7)$ & $114.65(4.26)$ & 0.44 \\
EDE-Q & $.99(1.46)$ & $0.36(.33)$ & 0.13 \\
BDI & $5.44(6.36)$ & $1.17(1.38)$ & 0.31 \\
Trait anxiety & $15.75(9.79)$ & $14.21(8.07)$ & 0.68 \\
State anxiety & $12.67(7.31)$ & $9.93(4.27)$ & 0.31 \\
Positive affect & $24.81(5.05)$ & $25.71(7.3)$ & 0.50 \\
Negative affect & $12.75(4.45)$ & $12.0(4.98)$ & 0.36 \\
RRS-ED & $16.5(7.03)$ & $10.15(1.52)$ & 0.006 \\
Duration of & $42(28.57)$ & - & - \\
$\quad$ AN (months) & & & - \\
Age of AN & $14.73(1.71)$ & - & \\
$\quad$ onset (years) & & & \\
\hline
\end{tabular}

BMI, body mass index; BDI-II, Beck Depression inventory; EDE-Q, Eating Disorder Examination questionnaire; RRS-ED, Ruminative Response Scale for eating disorders.

were evaluated further (Fig. 1, shows eight RSNs). The other components reflected distinct artifacts resulting from head motion and physiological or scanner noise. The RSNs of interest included: medial visual, lateral visual, auditory, sensory-motor, DMN, cognitive control, and fronto-parietal (right and left). These networks corresponded to RSNs which have been described previous and show high stability over time [Beckmann et al., 2005].

Significant between-group differences in the voxel-wise spatial distribution of the functional connectivity maps were subsequently revealed in the DMN. Significantly increased temporal correlation (coherence) was observed in recovered AN relative to healthy controls between the DMN functional connectivity map and in a region of the right precuneus close to the border of the posterior cingulate gyrus $(t=4.84$, BA31, $[8,-60,24])$ and the dorsolateral prefrontal cortex/inferior frontal gyrus (DLPFC/IFG) $(t=4.99$, BA9, [44, 6, 26]) (Figs. 2a,b and 3). Clusters were determined by using threshold-free cluster enhancement (TFCE) [Smith and Nichols, 2009] and an FWE corrected significance threshold of $P=0.05$ (i.e., fully corrected for multi-comparisons across voxels, within the DMN). These significant results would not survive further corrections for multiple comparisons, for example, correcting for all RSNs identified, or for two-tailed testing (i.e., the $\mathrm{HC}>$ recovered AN contrast). We believe that the primary RSN of relevance here would be expected to be the DMN (for example, see references and discussion below, with respect to existing literature on AN using task fMRI), and hence such further corrections are not necessary.

The lack of difference in the other RSNs identified (including purely visual and sensory-motor) argues 


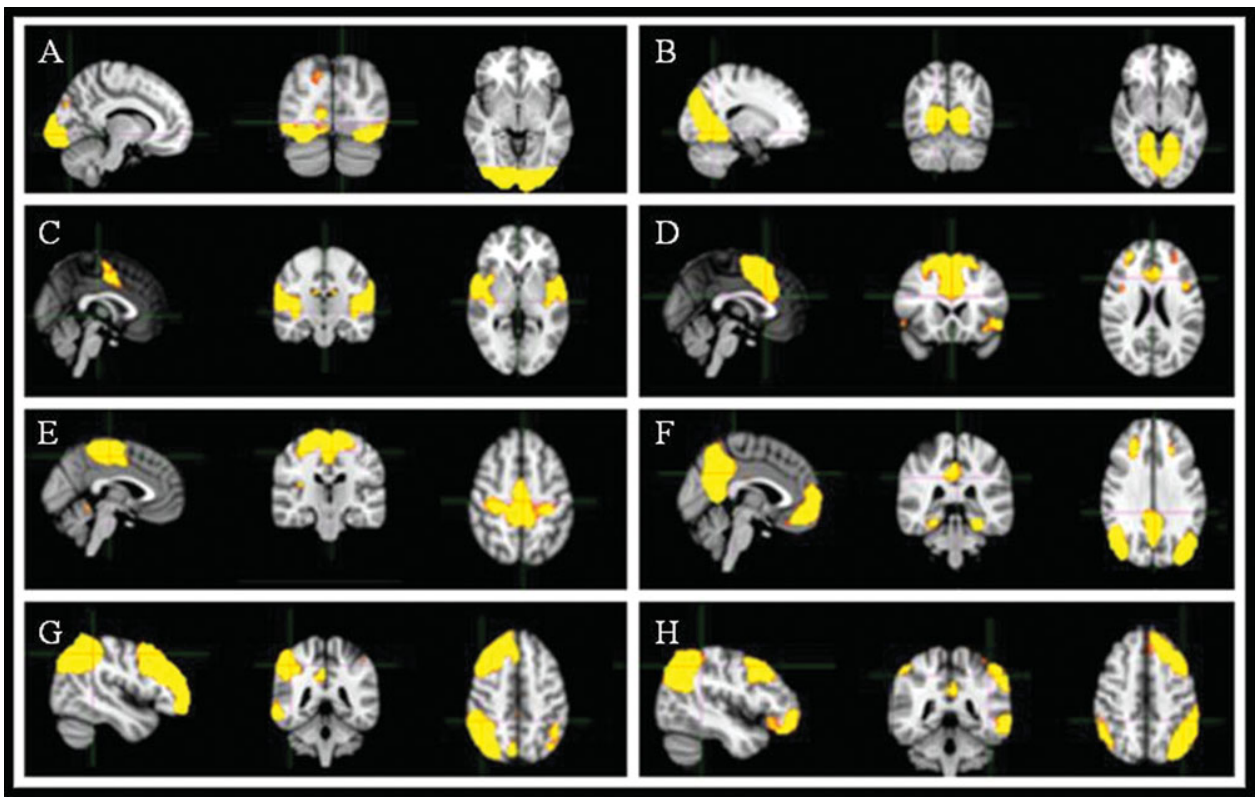

Figure I.

Sagittal, coronal and axial slices for the main RSNs detected, overlaid onto a standard EPI functional template. (A) lateral visual, (B) medial visual, (C) auditory, (D) cognitive control, (E) sensory-motor, (F) DMN, (G) left fronto-parietal, (H) right fronto-parietal.

Precuneus $(8,-60,24)$

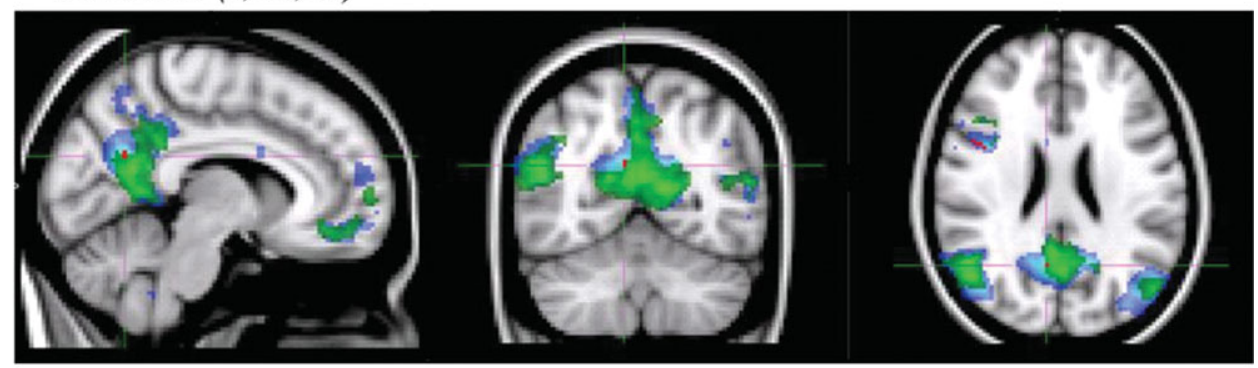

\section{DLPFC/IFG $(44,6,26)$}

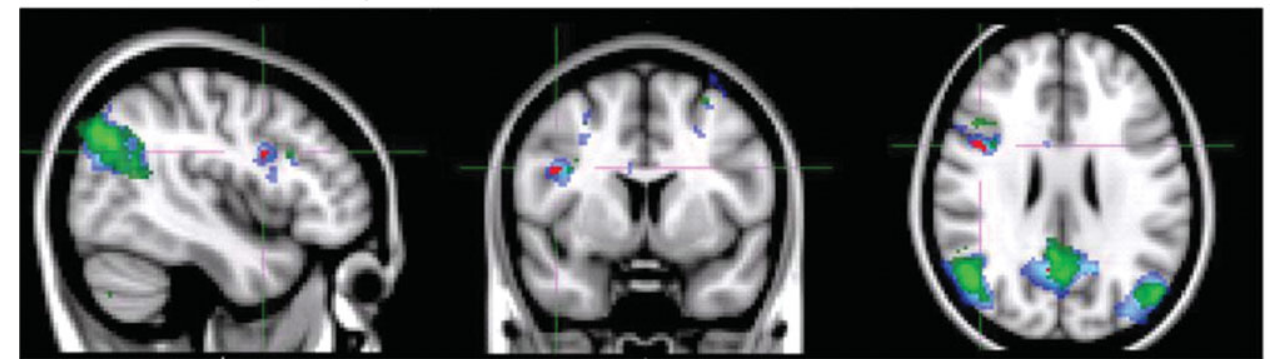

Figure 2.

Average DMN map from both groups combined and between-group differences. Networks are overlaid onto the MNI-I52 standard brain. $\square$, recovered AN group average DMN map $(Z>5)$; $\square$, healthy control group average DMN map $(Z>5)$; $\square$, group differences in functional connectivity between the DMN and the precuneus and the DLPFC/IFG: recovered AN > healthy controls, corrected $(P<0.05)$ (i.e., fully significant for multiple comparisons across voxels, within the DMN). 

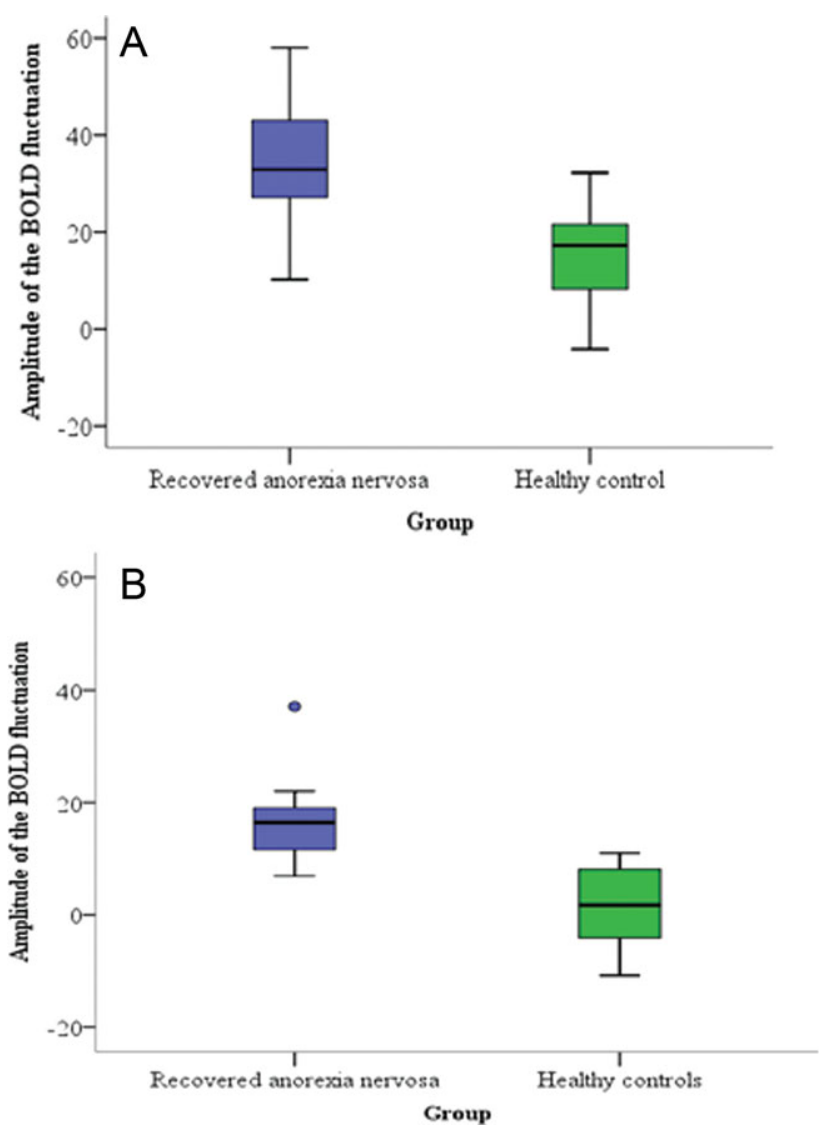

Figure 3.

Box plots show an increase in the amplitude of the BOLD fluctuation in the recovered anorexia nervosa participants compared to the healthy control participants between the DMN and the (A) precuneus $(8,-60,24)$ and, (B) DLPFC/IFG $(44,6,26)$. [Color figure can be viewed in the online issue, which is available at wileyonlinelibrary.com.]

against the DMN difference being caused by non-neural physiological group differences such as global perfusion or heart rate differences.

\section{Gray Matter Morphology}

No differences in gray matter were observed between the healthy controls and those recovered from AN. This indicates that the altered functional connectivity within the network discussed above is unlikely to be related to macroscopic (i.e. MRI observable) structural gray matter abnormalities.

\section{Correlations With Self-reported Rumination and ED Symptoms in AN}

Total scores on the RRS-ED, BDI-II and EDE-Q where examined for correlations with the precuneus and DLPFC dual regression output. Collapsing across subject group there was a positive correlation between the DLPFC output and the RRS-ED ( $r s=0.46, P=0.001)$ and EDE-Q ( $r s$ $=0.58, P=0.01)$ scores but there was no significant correlation with the BDI-II. There were no significant correlations between any of the questionnaire measures and the precuneus. When examining the two groups separately, there were no significant correlations.

\section{DISCUSSION}

This is the first study, to our knowledge, to examine whole brain resting state functional connectivity in participants who have recovered from AN. We found increased resting state functional connectivity between the DMN and the precuneus and DLPFC in those recovered from AN compared to healthy control participants. Our results support our hypothesis that resting state networks involving self-referential processing and cognitive control may be dysfunctional in AN and may serve as a neural biomarker for AN.

The precuneus has a pivotal role in the DMN and as such is involved in functions that are self-referential in nature [Greicius et al., 2009]. It has been posited that a decrease in DMN activity during an effortful task reflects the need to turn off self-referential processing to deploy attention to the demands of the task [Gusnard et al., 2001; Shulman et al., 1997].Thus, it may be that the increased functional connectivity in the precuneus in our data might underpin the rumination seen in AN. Disorder-specific rumination on eating, weight, and shape in AN [Cowdrey and Park, 2011, 2012; Park et al., 2011, 2012] has been discussed as an important maintaining factor in a recent framework for AN [Kaye et al., 2009; Park et al., 2011, 2012] and has some preliminary supporting evidence [Cowdrey and Park, 2011, 2012; Rawal et al., 2011; Wildes et al., 2010].

Recovered AN participants also showed significantly greater resting state functional connectivity in the DLPFC/ IFG compared to the healthy controls. The DLPFC is an important node in the cognitive control network [Cole and Schneider, 2007] and has been implicated in response inhibition [Swick et al., 2008], risk aversion [Christopoulos et al., 2009], and emotion control [Wang et al., 2008]. It is possible that our result of increased activity in the DLPFC area might contribute to the excessive inhibitory control seen in AN. Furthermore, as perseverative and obsessive neuropsychological profiles are reported in AN [Roberts et al., 2007; Zastrow et al., 2009], our results support the idea of an imbalance between top-down cognitive control and bottom-up appetitive processing in AN [Kaye et al., 2009]. Specifically, Kaye et al. [2009] propose that hyperactivity in the cognitive control circuit drives motivated behaviors and functions to control emotional response in AN, manifesting symptomatically as increased worry, perfectionism and concern with consequences. Task-based 
fMRI studies in both current and recovered AN participant's support this premise. Wagner et al. [2007] demonstrated an increased neural response in the DLPFC to both positive and negative feedback in a recovered AN sample and we have recently shown in a comparable sample an increased neural response in this region to an aversive taste [Cowdrey et al., 2011]. Similarly, Zastrow et al. [2009] used a set-shifting paradigm to assess cognitive-behavioral flexibility in participants with current AN and showed that AN participants were not only impaired on the task but that this was associated with increased neural activity in frontoparietal brain regions thus supporting the notion of increased cognitive control in AN. Our data adds to this literature by revealing increased DLPFC resting state functional connectivity in those who have had AN.

As the current study used a sample of individuals recovered from $\mathrm{AN}$, it is difficult to determine whether the increased functional connectivity effects are "scars" of previous illness episodes or premorbid risk factors which exist before illness onset. Longitudinal studies would be advantageous in order to confirm altered resting-state functional connectivity as a pre-morbid biomarker, although identifying those at risk of developing the disorder is challenging due to the relative low base rates of the illness and the young average age of onset [Rachelle and Lilenfled, 2010]. Replication of this study in those currently ill would however enable researchers to distinguish trait from state effects.

Interestingly, there have also been reports of increased resting state functional connectivity in the DMN in depression [Greicius et al., 2007; Sheline et al., 2010; Zhou et al., 2010] which authors have related to the increased selffocus and rumination seen in depression. As rumination and self-focus is also a negative feature of $\mathrm{AN}$, it is perhaps consistent that we also find increased functional connectivity in the DMN in the recovered AN group. However, it is also important to note that there are studies finding decreased DMN resting state functional connectivity in depression [Anand et al., 2005a; Bluhm, et al., 2009; Veer et al., 2010; Zhu et al., 2011]. The decreased DMN resting state functional connectivity in depression might pertain to disorder-specific features of depression and not AN such as depressed mood and poor concentration. Therefore, our results of increased DMN resting state functional connectivity in the AN group might be indicative of the increased self-focus, rumination and increased cognitive control in relation to eating and the body exhibited in AN.

\section{CONCLUSIONS}

We have demonstrated that participants with a history of AN have increased resting state functional connectivity between the DMN and the precuneus and the DLPFC/IFG. Our results are compatible with the core symptoms of AN including ruminative preoccupation on eating weight and shape, excessive planning and impaired cognitive flexibility
[Kaye et al., 2009; Park et al., 2011, 2012]. Considering the advantages of resting state for clinical populations (good signal to noise BOLD, minimal patient compliance), future research employing longitudinal designs should be conducted. We believe by examining individuals at risk of developing AN, through the illness course and into recovery, will aid the discovery of viable neural network biomarkers for psychiatric disorders such as AN.

\section{REFERENCES}

American Psychiatric Association (1994): Diagnostic and Statistical Manual of Mental Health Disorders. Washington, DC: American Psychiatric Publishing.

Anand A, Li Y, Wang Y, Wu J, Gao S, Bukhari L, Mathews VP, Kalnin A, Lowe MJ (2005a): Activity and connectivity of brain mood regulating circuit in depression: A functional magnetic resonance study. Biol Psychiatry 57:1079-1088.

Anand A, Li Y, Wang Y, Wu J, Gao S, Bukhari L, Mathews VP, Kalnin A, Lowe MJ (2005b): Antidepressant effect on connectivity of the mood-regulating circuit: An fMRI study. Neuropsychopharmacology 30:1334-1344.

Arcelus J, Mitchell AJ, Wales J, Nielsen S (2011): Mortality rates in patients with anorexia nervosa and other eating disorders: A meta-analysis of 36 studies. Arch. Gen. Psychiatry 68:724-731.

Beck A, Steer R, Brown G (2002): BDI II Manual. San Antonio, Texas: Psychological Corp.

Beckmann CF, DeLuca M, Devlin JT, Smith SM (2005): Investigations into resting-state connectivity using independent component analysis. Philos Trans Roy Soc B: Biol Sci 360:1001-1013.

Berman MG, Peltier S, Nee DE, Kross E, Deldin PJ, Jonides J (2010): Depression, rumination and the default network. Social Cogn Affect Neurosci 6:548-555.

Biswal BB, Mennes M, Zuo X-N, Gohel S, Kelly C, Smith SM, Beckmann CF, Adelstein JS, Buckner RL, Colcombe S, Dogonowski A-M, Ernst M, Fair D, Hampson M, Hoptman MJ, Hyde JS, Kiviniemi VJ, Kotter R, Li S-J, Lin C-P, Lowe MJ, Mackay C, Madden DJ, Madsen KH, Margulies DS, Mayberg HS, McMahon K, Monk CS, Mostofsky SH, Nagel BJ, Pekar JJ, Peltier SJ, Petersen SE, Riedl V, Rombouts SARB, Rypma B, Schlaggar BL, Schmidt S, Seidler RD, Siegle GJ, Sorg C, Teng G-J, Veijola J, Villringer A, Walter M, Wang L, Weng X-C, Whitfield-Gabrieli S, Williamson $\mathrm{P}$, Windischberger C, Zang Y-F, Zhang H-Y, Castellanos FX, Milham MP (2010): Toward discovery science of human brain function. Proc Natl Acad Sci 107:4734-4739.

Bluhm R, Williamson P, Lanius R, Théberge J, Densmore M, Bartha R, Neufeld R, Osuch E (2009): Resting state default-mode network connectivity in early depression using a seed regionof-interest analysis: Decreased connectivity with caudate nucleus. Psychiatry Clin Neurosci 63:754-761.

Castro-Fornieles J, Bargalló N, Lázaro L, Andrés S, Falcon C, Plana MT, Junqué C (2009): A cross-sectional and follow-up voxel-based morphometric MRI study in adolescent anorexia nervosa. J Psychiatric Res 43:331-340.

Christopoulos GI, Tobler PN, Bossaerts P, Dolan RJ, Schultz W (2009): Neural correlates of value, risk, and risk aversion contributing to decision making under risk. J Neurosci 29:12574-12583.

Cole MW, Schneider W (2007): The cognitive control network: Integrated cortical regions with dissociable functions. Neuroimage 37:343-360. 
Cowdrey FA, Park RJ (2011): Assessing rumination in eating disorders: Principal component analysis of a minimally modified ruminative response scale. Eating Behav 12:321-324.

Cowdrey FA, Park RJ (2012): The role of experiential avoidance, rumination and mindfulness in eating disorders. Eating Behav 13:100-105.

Cowdrey FA, Park RJ, Harmer CJ, McCabe C (2011): Increased neural processing of rewarding and aversive food stimuli in recovered anorexia nervosa. Biol Psychiatry 70:736-743.

Cowdrey FA, Harmer CJ, Park RJ, McCabe C (2012): Neural responses to emotional faces in women recovered from anorexia nervosa. Psychiatry Res 201:190-195

Damoiseaux JS, Rombouts SARB, Barkhof F, Scheltens P, Stam CJ, Smith SM, Beckmann CF (2006): Consistent resting-state networks across healthy subjects. Proc Natl Acad Sci 103:13848-13853.

Douaud Gl, Smith S, Jenkinson M, Behrens T, Johansen-Berg H, Vickers J, James S, Voets N, Watkins K, Matthews PM, James A (2007): Anatomically related grey and white matter abnormalities in adolescent-onset schizophrenia. Brain 130:2375-2386.

Fairburn CG (2005): Evidence-based treatment of anorexia nervosa. Int J Eating Disord 37(S1):S26-S30.

Fairburn CG, Beglin SJ (2008): Eating disorder examination questionnaire (EDE-Q 6.0). In: Fairburn C, editor. Cognitive Behaviour Therapy and Eating Disorders. New York: Guildford Press. pp 309-313.

Filippini N, MacIntosh BJ, Hough MG, Goodwin GM, Frisoni GB, Smith SM, Matthews PM, Beckmann CF, Mackay CE (2009): Distinct patterns of brain activity in young carriers of the APOE- $\varepsilon 4$ allele. Proc Natl Acad Sci 106:7209-7214.

Greicius MD (2008): Resting-state functional connectivity in neuropsychiatric disorders. Curr Opin Neurol 21:424-430.

Greicius MD, Flores BH, Menon V, Glover GH, Solvason HB, Kenna H, Reiss AL, Schatzberg AF (2007): Resting-state functional connectivity in major depression: Abnormally increased contributions from subgenual cingulate cortex and thalamus. Biol Psychiatry 62:429-437.

Greicius MD, Supekar K, Menon V, Dougherty RF (2009): Restingstate functional connectivity reflects structural connectivity in the default mode network. Cereb Cortex 19:72-78.

Gusnard DA, Raichle ME (2001): Searching for a baseline: Functional imaging and the resting human brain. Nat Rev Neurosci 2:685-694.

Gusnard DA, Akbudak E, Shulman GL, Raichle ME (2001): Medial prefrontal cortex and self-referential mental activity: Relation to a default mode of brain function. Proc Natl Acad Sci 98:4259-4264.

Hamilton JP, Furman DJ, Chang C, Thomason ME, Dennis E, Gotlib IH (2010): Default-mode and task-positive network activity in major depressive disorder: Implications for adaptive and maladaptive rumination. Biol Psychiatry 70:327-333.

Hayasaka S, Phan KL, Liberzon I, Worsley KJ, Nichols TE (2004): Nonstationary cluster-size inference with random field and permutation methods. Neuroimage 22:676-687.

Jacobs MJ, Roesch S, Wonderlich SA, Crosby R, Thornton L, Wilfley DE, Berrettini WH, Brandt $\mathrm{H}$, Crawford S, Fichter MM, Halmi KA, Johnson C, Kaplan AS, LaVia M, Mitchell JE, Rotondo A, Strober M, Woodside DB, Kaye WH, Bulik CM (2009): Anorexia nervosa trios: Behavioral profiles of individuals with anorexia nervosa and their parents. Psychol Med 39:451-461.

Jenkinson M, Beckmann CF, Behrens TEJ, Woolrich MW, Smith SM (2012): FSL. Neuroimage 62:782-790.
Joos A, Hartmann A, Glauche V, Perlov E, Unterbrink T, Saum B, Tüscher O, Tebartz van Elst L, Zeeck A (2011): Grey matter deficit in long-term recovered anorexia nervosa patients. Eur Eating Disord Rev 19:59-63.

Katzman DK, Zipursky RB, Lambe EK, Mikulis DJ (1997): A longitudinal magnetic resonance imaging study of brain changes in adolescents with anorexia nervosa. Arch Pediatr Adolesc Med 151:793-797.

Kaye WH, Fudge JL, Paulus M (2009): New insights into symptoms and neurocircuit function of anorexia nervosa. Nat Rev Neurosci 10:573-584.

McCabe C, Mishor Z (2011): Antidepressant medications reduce subcortical-cortical resting-state functional connectivity in healthy volunteers. Neuroimage 57:1317-1323.

McCabe C, Mishor Z, Filippini N, Cowen PJ, Taylor MJ, Harmer CJ (2011): SSRI administration reduces resting state functional connectivity in dorso-medial prefrontal cortex. Mol Psychiatry 16:592-594.

Nichols TE, Holmes AP (2002): Nonparametric permutation tests for functional neuroimaging: A primer with examples. Hum Brain Map 15:1-25.

Park RJ, Dunn BD, Barnard PJ (2011): Schematic models and modes of mind in anorexia nervosa. I. A novel process account. Int J Cogn Therapy 4:415-437.

Park RJ, Dunn BD, Barnard PJ (2012): Schematic models and modes of mind in anorexia nervosa. II. Implications for treatment and course. Int J Cogn Therapy 5:86-98.

Rachelle L, Lilenfled R (2010): Personality and temperament. In: Adan R, Kaye W, editors. Behavioural Neurobiology of Eating Disorders, 1st ed. Berlin: Springer. pp 4-13.

Raichle ME, MacLeod AM, Snyder AZ, Powers WJ, Gusnard DA, Shulman GL (2001): A default mode of brain function. Proc Natl Acad Sci 98:676-682.

Rawal A, Williams JMG, Park RJ (2011): Effects of analytical and experiential self-focus on stress-induced cognitive reactivity in eating disorder psychopathology. Behav Res Ther 49:635-645.

Roberto CA, Mayer LES, Brickman AM, Barnes A, Muraskin J, Yeung L-K, Steffener J, Sy M, Hirsch J, Stern Y, Walsh BT (2011): Brain tissue volume changes following weight gain in adults with anorexia nervosa. Int J Eating Disord 44:406-411.

Roberts M, Tchanturia K, Stahl D, Southgate L, Treasure J (2007): A systematic review and meta-analysis of set-shifting ability in eating disorders. Psychol Med 37:1075-1084.

Sheline YI, Price JL, Yan Z, Mintun MA (2010): Resting-state functional MRI in depression unmasks increased connectivity between networks via the dorsal nexus. Proc Natl Acad Sci 107:11020-11025.

Shulman GL, Fiez JA, Corbetta M, Buckner RL, Miezin FM, Raichle ME, Petersen SE (1997): Common blood flow changes across visual tasks. II. Decreases in cerebral cortex. J Cogn Neurosci 9:648-663.

Smith SM, Nichols TE (2009): Threshold-free cluster enhancement: Addressing problems of smoothing, threshold dependence and localisation in cluster inference. Neuroimage 44:83-98.

Spielberger C, Gorsuch, RC, Lushene RE, Vagg PR, Jacobs GA (1983): Manual for the State-Trait Anxiety Inventory. Palo Alto: Consulting Psychologist Press.

Spitzer R, Williams J, Gibbon M, First M (2004): Structured Clinical Interview for the DSM-IV (SCID-I/P). Arlington, VA: American Psychiatric Press.

Sullivan P (1995): Mortality in anorexia nervosa. Am J Psychiatry 152:1073-1074. 
Swick D, Ashley V, Turken A (2008): Left inferior frontal gyrus is critical for response inhibition. BMC Neurosci 9:102.

Uher R, Murphy T, Brammer MJ, Dalgleish T, Phillips ML, Ng VW, Andrew CM, Williams SC, Campbell IC, Treasure J (2004): Medial prefrontal cortex activity associated with symptom provocation in eating disorders. Am J Psychiatry 161:1238-1246.

van Kuyck K, Gérard N, Laere KV, Casteels C, Pieters G, Gabriëls L, Nuttin B (2009): Towards a neurocircuitry in anorexia nervosa: Evidence from functional neuroimaging studies. J Psychiatric Res 43:1133-1145.

Veer IM, Beckmann C, Van Tol M-J, Ferrarini L, Milles J, Veltman D, Aleman A, Van Buchem MA, Van Der Wee NJA, Rombouts SAR (2010): Whole brain resting-state analysis reveals decreased functional connectivity in major depression. Front Syst Neurosci 4:1-10.

Wagner A, Aizenstein H, Venkatraman VK, Fudge J, May JC, Mazurkewicz L, Frank GK, Bailer UF, Fischer L, Nguyen V, Carter C, Putnam K, Kaye WH (2007): Altered reward processing in women recovered from anorexia nervosa. Am J Psychiatry 164:1842-1849.

Wagner A, Barbarich-Marsteller NC, Frank GK, Bailer UF, Wonderlich SA, Crosby RD, Henry SE, Vogel V, Plotnicov K, McConaha C, Kaye WH (2006a): Personality traits after recovery from eating disorders: Do subtypes differ? Int J Eating Disord 39:276-284.
Wagner A, Greer P, Bailer UF, Frank GK, Henry SE, Putnam K, Meltzer CC, Ziolko SK, Hoge J, McConaha C, Kaye WH (2006b): Normal brain tissue volumes after long-term recovery in anorexia and bulimia nervosa. Biol Psychiatry 59:291-293.

Wang L, LaBar KS, Smoski M, Rosenthal MZ, Dolcos F, Lynch TR, Krishnan RR, McCarthy G (2008): Prefrontal mechanisms for executive control over emotional distraction are altered in major depression. Psychiatry Res Neuroimaging 163:143-155.

Watson D, Clark L, Tellegen A (1988): Development and validation of brief measures of positive and negative affect: The PANAS scales. J Person Social Psychol 54:1063-1070.

Wildes JE, Ringham RM, Marcus MD (2010): Emotion avoidance in patients with anorexia nervosa: Initial test of a functional model. Int J Eating Disord 43:398-404.

Zastrow A, Kaiser, Stefan, Stippich C, Walther S, Herzog W, Tchanturia K, Belger A, Weisbrod M, Treasure J, Friederich HC (2009): Neural correlates of impaired cognitive-behavioral flexibility in anorexia nervosa. Am J Psychiatry 166:608-616.

Zhou Y, Yu C, Zheng H, Liu Y, Song M, Qin W, Li K, Jiang T (2010): Increased neural resources recruitment in the intrinsic organization in major depression. J Affect Disord 121:220-230.

Zhu X, Wang X, Xiao J, Liao J, Zhong M, Wang W, Yao S (2012): Evidence of a dissociation pattern in resting-state default mode network connectivity in first-episode, treatment-naive major depression patients. Biol Psychiatry 611-617. 